\title{
T-Center: A Novel Discriminative Feature Extraction Approach for Iris Recognition
}

\author{
Yifeng Chen, ${ }^{1}$ Cheng Wu, ${ }^{2}$ Yiming Wang ${ }^{3}$ \\ School of Rail Transportation, Soochow University \\ yfchen@stu.suda.edn.cn, cwu@ suda.edu.cn, ymwang@ suda.edu.cn
}

\begin{abstract}
For large-scale iris recognition tasks, the determination of classification thresholds remains a challenging task, especially in practical applications where sample space is growing rapidly. Due to the complexity of iris samples, the classification threshold is difficult to determine with the increase of samples. The key issue to solving such threshold determination problems is to obtain iris feature vectors with more obvious discrimination. Therefore, we train deep convolutional neural networks based on a large number of iris samples to extract iris features. More importantly, an optimized center loss function referred to Tight Center $(\mathcal{T}$-Center $)$ Loss is used to solve the problem of insufficient discrimination caused by Softmax loss function. In order to evaluate the effectiveness of our proposed method, we use cosine similarity to estimate the similarity between the features on the published datasets CASIA-IrisV4 and IITD2.0. Our experiment results demonstrate that the $\mathcal{T}$-Center loss can minimize intra-class variance and maximize inter-class variance, which achieve significant performance on the benchmark experiments.
\end{abstract}

\section{Introduction}

Iris recognition is one of the most promising fields in biometrics. The first complete iris recognition system was presented by Daugman (Daugman 1993). Over the past few years, conventional iris recognition under homogeneous and controlled conditions has been extensively studied. The general procedures for iris recognition include four parts: iris location, iris segmentation, feature encoding and feature matching. Recently, more attention has been paid on the realization of large-scale iris recognition tasks with massive sample space. Unfortunately, the non-ideal captured images increase the difficulty of iris segmentation and cross-device picture sources lead to adaptive trapping of parameters.

Recent progress in deep learning, in particular, deep Convolutional Neural Networks (CNNs) have significantly improved the state-of-the-art performance for computer vision tasks, which makes deep CNNs a dominant machine learning approach for computer vision. Deep learning based approaches in iris biometrics, have been explored in recent works. Liu proposed a deep framework for iris verification,

Copyright (c) 2019, Association for the Advancement of Artificial Intelligence (www.aaai.org). All rights reserved. which learns relational features to measure the similarity between pairs of iris images (Liu et al. 2016). Gangwar (Gangwar and Joshi 2016) proposed a deep learning based method for iris learning and various optimal tricks were used to avoid overfitting. These typical researches have an interesting common point: all deep models are trained under the supervision of the Softmax loss. The advantage of Softmax loss is to make the learned features highly separable, but it does not guarantee the features' high discrimination. Unfortunately, higher discrimination is the key to robustly identify new unseen classes without label prediction.

Thus, we propose a novel $\mathcal{T}$-Center loss in order to enhance the discriminative power of the deeply learned features. The original center loss function fuse the Euclidean distance between the features and features centers into loss function to maximize the inter-class variance and minimize the intra-class variance (Wen et al. 2016), which is essentially a kind of distance metric learning. However for iris recognition task, we care more about the angular metric rather than distance metric since cosine distance between two features is used to compute the similarity score.

\section{$\mathcal{T}$-Center loss}

Deep learning uses the loss function to measure the degree of optimization and it is clear that designing an appropriate loss function can enhance the ability of discrimination. The most commonly used loss function is Softmax loss. Assuming that the input learned feature vectors $x_{i}$ and its label $y_{i}$, the original Softmax loss function is as Equation: $L_{s}=-\sum_{i=1}^{m} \log \frac{e^{W_{y_{i}}^{T} x_{i}+b_{y_{i}}}}{\sum_{j=1}^{n} e^{W_{j}^{T} x_{i}+b_{j}}}$ where $m$ is the size of a mini-batch, $n$ the number of classes and $f_{j}$ the $j$-th element of the class score vector $f$. Here $j \in[1, n]$. In typical CNNs, $f$ is denoted as the output of the FC layers, we have $f_{j}=W_{j}^{T} x_{i}+b_{j}$ and $f_{y_{i}}=W_{y_{i}}^{T} x_{i}+b_{y_{i}}$, where $W_{j}$ and $W_{y_{i}}$ denote the weights of the last fully connected layer corresponding to the class $j$ and $y_{i}$ respectively.

However, for iris recognition task, the deep features learned under Softmax loss are not separable enough to discriminate the close-set iris samples. To enhance the discriminative power of the features, we propose the novel $\mathcal{T}$-Center loss function. $L_{t c}=\frac{1}{2} \sum_{i=1}^{m}\left\|x_{i}^{*}-c_{y_{i}}^{*}\right\|^{2}$ where $c_{y_{i}}$ denotes the center of the deep feature corresponding to 


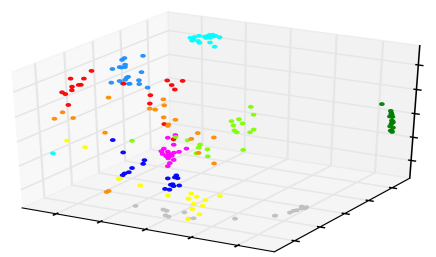

(a) Model A: Softmax scatter

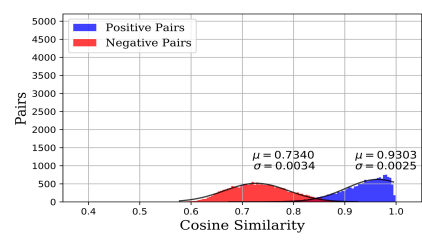

(d) Model A: Softmax histogram

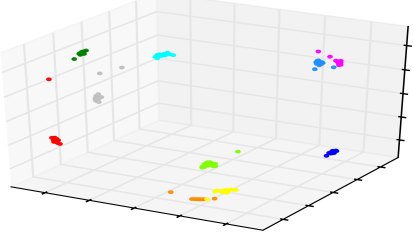

(b) Model B: Softmax+Center

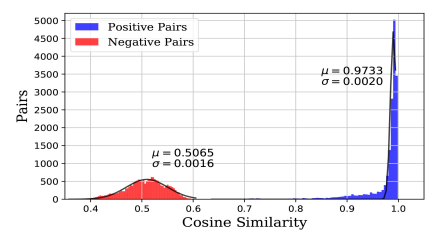

(e) Model B: Softmax+Center

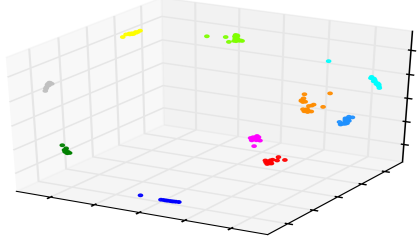

(c) Model C: Softmax $+\mathcal{T}$-Center

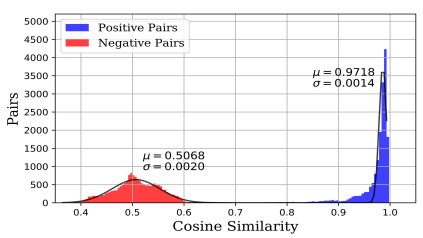

(f) Model C: Softmax $+\mathcal{T}$-Center

Figure 1: Visualization of features learned under different loss functions. The first column shows the distributions of 3D features in Euclidean space after PCA. The points with different colors denote the features from different classes. The second column shows the cosine similarity distributions of both positive pairs and negative pairs (19821 images, 500 classes).

the $y_{i}$-th class. In our opinion, the simple Euclidean margin $\left\|x_{i}-c_{y_{i}}\right\|$ is not always suitable for learning more discriminative iris features, so that $c_{y_{i}}^{*}$ and $x_{i}^{*}$ denote the feature center and feature vector after L2-norm respectively. In instead of updating the feature centers by back propagation algorithm, the centers are computed by averaging the features of the corresponding classes in each iteration. The update equation of $c_{y_{i}}$ is computed using equation: $\Delta c_{j}^{*}=\frac{\sum_{i=1}^{m} \delta\left(y_{i}=j\right) \cdot\left(c_{j}^{*}-x_{i}^{*}\right)}{1+\sum_{i=1}^{m} \delta\left(y_{i}=j\right)}, c_{j}^{*^{t+1}}=c_{j}^{*^{t}}-\alpha \cdot \Delta c_{j}^{*^{t}}$ where $t$ is the number of iteration. The feature centers update only if $y_{i}$ and $j$ share the same class, where $\delta=1$. A scalar $\alpha$ is used to control the learning rate of the centers to avoid large perturbations caused by image noise.

Note that these learned features and centers would degrade to zeros when the $\mathcal{T}$-Center loss is used separately for deep networks. So it is necessary to adopt the joint supervision of Softmax loss and $\mathcal{T}$-Center loss to train. The final loss function is given as $L=-\sum_{i=1}^{m} \log \frac{e^{W_{y_{i}}^{T} x_{i}+b_{y_{i}}}}{\sum_{j=1}^{n} e^{W_{j}^{T} x_{i}+b_{j}}}+$ $\frac{\lambda}{2} \sum_{i=1}^{m}\left\|x_{i}^{*}-c_{y_{i}}^{*}\right\|^{2}$, where $\lambda$ is a scalar used for balancing the significance of two loss functions.

\section{Effect of CNNs architectures}

In order to visually demonstrate the ability of the proposed method to distinguish iris feature vectors, we illustrate a toy example containing 10 classes. Figure 1 gives the Euclidean feature space distribution and the cosine similarity of both positive pairs and negative pairs using different loss functions. It is empirically demonstrate that compared with Model A (supervised by Softmax) and Model B (supervised jointly by Softmax and center loss), Model C (supervised jointly by Softmax and $\mathcal{T}$-Center loss) has $43.8 \%$ and $30.2 \%$ decrease on the intra-class variance respectively. It indicates that using the $\mathcal{T}$-Center loss results in a more discriminative distribution in the feature space and a larger angular margin, which is very suitable for large-scale iris recognition tasks.
Table 1: Variance of positive and negative pairs

\begin{tabular}{lcc}
\hline Method & Positive variance $\left(\times 10^{-3}\right)$ & Negative variance $\left(\times 10^{-3}\right)$ \\
\hline Model A & 2.51 & 3.42 \\
Model B & 2.02 & 1.61 \\
Model C & $\mathbf{1 . 4 1}$ & $\mathbf{1 . 9 8}$ \\
\hline
\end{tabular}

\section{Conclusion}

Large-scale iris recognition tasks remains a challenging task in practical applications due to the difficulty of classification threshold determination. The paper proposes a novel feature extraction approach for iris recognition, which can obtain iris feature vectors with more obvious discrimination. We refer the novel loss function to $\mathcal{T}$-Center loss. Jointly supervised by the linear combination of $\mathcal{T}$-Center loss and softmax, the discriminative power of the deep features based on CNNs can be highly enhanced.

\section{References}

Daugman, J. G. 1993. High confidence visual recognition of persons by a test of statistical independence. IEEE transactions on pattern analysis and machine intelligence 15(11):1148-1161.

Gangwar, A., and Joshi, A. 2016. Deepirisnet: Deep iris representation with applications in iris recognition and crosssensor iris recognition. In Image Processing (ICIP), 2016 IEEE International Conference on, 2301-2305. IEEE.

Liu, N.; Zhang, M.; Sun, Z.; and Tan, T. 2016. Deepiris: Learning pairwise filter bank for heterogeneous iris verification. Pattern Recognition Letters 82:154-161.

Wen, Y.; Zhang, K.; Li, Z.; and Qiao, Y. 2016. A discriminative feature learning approach for deep face recognition. In European Conference on Computer Vision, 499515. Springer. 